\title{
Medical Image of the Week: Wide Mediastinum Due to Lung Infiltrates
}

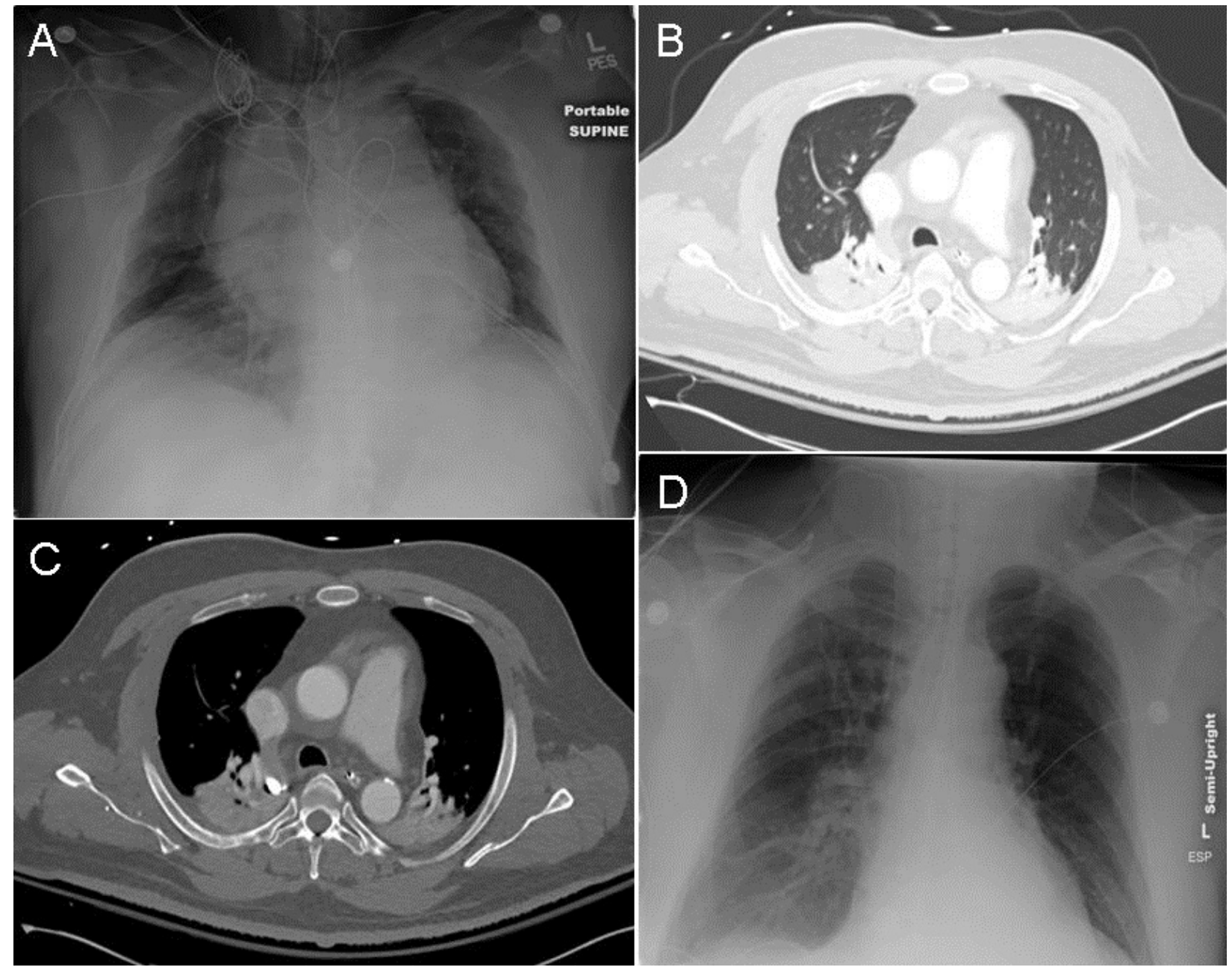

Figure 1. Panel A: Admission chest $\mathrm{x}$-ray showing an apparent widened mediastinum. Panels B \& C: CT scan showing consolidation in the posterior lungs bilaterally. Panel D: Chest $\mathrm{X}$-ray showing resolution of his widened mediastinum with an increase in positive end-expiratory pressure.

The patient is a 65-year-old man patient with a past medical history of poorly controlled hypertension and coronary artery disease who was admitted after a witnessed code arrest. He was found down, and paramedics arrived within 5 minutes and started chest compressions. His initial CXR showed a wide mediastinum (Figure 1A) that was concerning for possible aortic dissection especially with his history of poorly controlled hypertension. Due to his hemodynamic instability a chest CT scan couldn't be done initially, but the patient underwent a trans-esophageal echo that was negative for aortic dissection.

When the patient became more stable a chest CT scan with contrast was done and showed consolidation of the medial parts of both lungs with 7 bilateral rib fractures 
(Figure $1 \mathrm{~B} \& \mathrm{C}$ ). The impression was either lung contusion from the aggressive chest compression as evidenced by the bilateral 7 rib fractures or aspiration in the dependent parts of the lung. His apparent widened mediastinum resolved with increasing the positive end-expiratory pressure (PEEP) on the ventilator within 48 hours (Figure 1D).

Huthayfa Ateeli MBBS, Laila Abu Zaid MD

Department of Medicine

University of Arizona

Tucson, AZ.

\section{References}

1. Cohn SM. Pulmonary contusion: review of the clinical entity. J Trauma. 1997;42(5):973-9. [CrossRef] [PubMed]

2. Lai CC, Wang $\mathrm{CY}$, Lin HI, Wang JY. Pulmonary contusion associated with chest compressions. Resuscitation. 2010;81(1):133. [CrossRef] [PubMed] 